\title{
Convection in a Box of Porous Material Saturated with Fluid
}

\author{
J. L. BECK \\ Physics and Engineering Laboratory, Department of Scientific and Industrial Research, Lower Hutt, New Zealand
}

(Received 21 December 1971; final manuscript received 29 February 1972)

\begin{abstract}
The true critical Rayleigh number for the onset of a convective flow of a fluid in a rectangular box of porous material heated from below is found for various box geometries. The preferred cellular mode of the motion at Rayleigh numbers just above the critical is determined. In contrast with the established results for the similar problem in a continuous fluid, the roll (a cell with only two nonzero velocity components) is not the only cellular mode and the roll axis direction is such that there is the greatest degree of "squareness" in the cross section of each roll. The invalidity of a frequently used form of Darcy's law and the present form of the energy method for the stability of flows in which fluid crosses the boundaries is discussed.
\end{abstract}

\section{INTRODUCTION}

Studies associated with fluid flows in the ground below the thermal areas in many parts of the world, and in particular, in the North Island of New Zealand, indicate that convection plays an important role in both the fluid and heat transfer processes associated with such regions. It is thus of considerable interest to determine just when the fluid in a finite permeable region heated from below might be unstable (convecting). This paper arises from a section of this study.

Most of the work on the onset of convection in a porous medium is based on linear theory (see, for example, the review in the paper by Nield $^{1}$ ). The critical Rayleigh number derived by such a theory gives a necessary condition for stability (or, equivalently, a sufficient condition for instability). In contrast, the energy method for convective instability, developed by Joseph ${ }^{2}$ for continuous fluids and subsequently modified by Westbrook" for fluids in porous media, gives a critical Rayleigh number which provides a sufficient condition for stability of a motionless fluid. This paper examines the effect of lateral walls on the stability of a fluid saturating a porous medium heated from below using both of the above approaches. A true critical Rayleigh number is shown to be obtained. Diagrams are presented which make it possible, when the dimensions of the box of porous naterial are given, to determine the corresponding critical Rayleigh number and the preferred cellular mode at Rayleigh numbers just above the critical.

An analysis in the appendix illustrates the invalidity of the inclusion of the nonlinear inertia term, of the form taken by Lapwood, ${ }^{4}$ in Darcy's law. The energy method in its present form is thus inapplicable to a range of problems associated with fluid flows in porous media. The approach is, however, shown to be valid for initially stationary fluids.

\section{THE GOVERNING EQUATIONS}

Assuming rectangular axes with $O X$ and $O Y$ horizontal and $0 Z$ vertically downward, the walls of the box are taken to be at $X= \pm \frac{1}{2} a_{1}, Y= \pm \frac{1}{2} a_{2}$, and $Z=0, h$.
The vertical side walls are assumed to be thermally insulated while the upper and lower surfaces are maintained at the constant temperatures $T_{0}$ and $T_{1}$, respectively, $\left(T_{1}>T_{0}\right)$. All boundaries are assumed to be impermeable to the contained fluid.

Under the Boussinesq approximation, the governing equations are taken to be

Darcy's law:

$$
\epsilon^{-1} \frac{\partial \mathrm{q}}{\partial t}=\left[1-\gamma\left(T-T_{0}\right)\right] g \mathbf{k}-\rho_{0}^{-1} \nabla P-\frac{\nu}{k} \mathbf{q},
$$

heat conservation:

$$
(c \rho)_{m} \frac{\partial T}{\partial t}+c \rho_{0} q \cdot \nabla T=K_{m} \nabla^{2} T,
$$

where

continuity:

$$
(c \rho)_{m}=(1-\epsilon)(c \rho)_{8}+\epsilon c \rho_{0} .
$$

$$
\nabla \cdot q=0,
$$

where $\mathrm{q}, T$, and $P$ are the macroscopic fluid velocity, temperature, and pressure, respectively.

Here $(c \rho)_{m},(c \rho)_{s}$, and $c \rho_{0}$, are the heat capacities per unit volume of the saturated medium, the solid material making up the porous medium, and the fluid, respectively; $g$ is the gravitational acceleration; $k$ is the permeability of the medium; $K_{m}$ is the thermal conductivity of the saturated medium; $\gamma$ is the volumetric coefficient of thermal expansion of the fluid; $\epsilon$ is the porosity of the medium; $\rho_{0}$ is the density of the fluid [the (linear) density variation with temperature in the critical body-force term having been allowed for in (1)]; $\nu$ is the kinematic viscosity of the fluid; and $\mathbf{k}$ is a unit vector in the $0 Z$ direction. All the above quantities are assumed constant.

The problem is to investigate the stability of the basic state $\left(\mathbf{q}^{*}, T^{*}\right)$,

$$
\mathbf{q}^{*}=(0,0,0), \quad T^{*}=T_{0}+\left(T_{1}-T_{0}\right)(Z / h),
$$

to any disturbance $\left(q^{\prime}, T^{\prime}\right)$ giving a temperature and flow distribution satisfying the same boundary conditions as the basic state. 


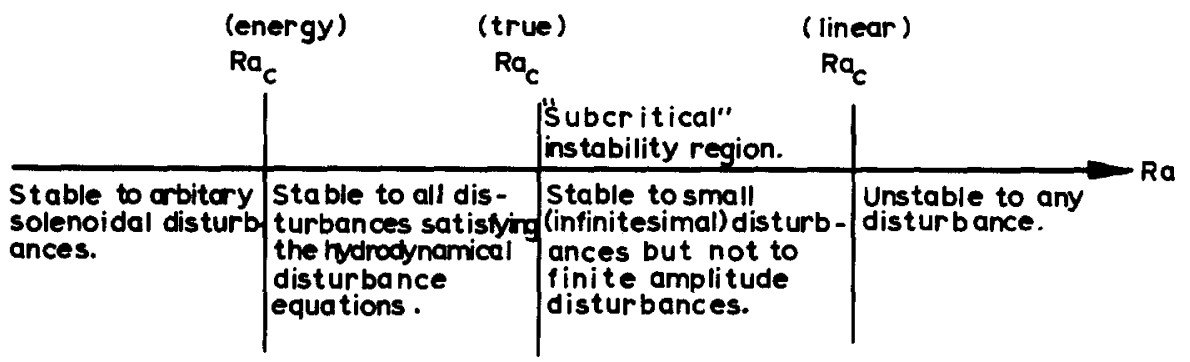

FIG. 1. Schematic diagram of the relationship between the various critical Rayleigh numbers.

The equations governing such a disturbance are

$$
\begin{gathered}
\epsilon^{-1} \frac{\partial \mathbf{q}^{\prime}}{\partial t}=-\gamma g T^{\prime} \mathbf{k}-\rho_{0}{ }^{-1} \nabla p^{\prime}-\frac{\nu}{k} \mathbf{q}^{\prime}, \\
(c \rho)_{m} \frac{\partial T^{\prime}}{\partial t}+c \rho_{0} \mathbf{q}^{\prime} \cdot \nabla T^{\prime}+c \rho_{0} \mathbf{q}^{\prime} \cdot \nabla T^{*}=K_{m} \nabla^{2} T^{\prime}, \\
\nabla \cdot \mathbf{q}^{\prime}=0,
\end{gathered}
$$

with$$
\mathbf{q}^{\prime} \cdot \mathbf{n}=0 \quad \text { on } X= \pm \frac{1}{2} a_{1}, \quad Y= \pm \frac{1}{2} a_{2}, \quad Z=0, h
$$

( $\mathbf{n}$ is the unit outward normal to the boundary surface) and

$$
\begin{gathered}
T^{\prime}=0 \quad \text { on } Z=0, h, \\
\frac{\partial T^{\prime}}{\partial n}=0 \quad \text { on } X= \pm \frac{1}{2} a_{1}, \quad Y= \pm \frac{1}{2} a_{2} .
\end{gathered}
$$

Here, $p^{\prime}$ is the pressure disturbance corresponding to the disturbance $\left(\mathbf{q}^{\prime}, T^{\prime}\right)$.

Equations (5), (6), and (7) and the boundary conditions (8) are reduced to dimensionless form by the substitutions:

$$
\begin{aligned}
& \mathbf{q}^{\prime}=\mathrm{Ra}^{1 / 2}(\kappa / h) \mathbf{v}, \quad T^{\prime}=\beta h \theta, \\
& p^{\prime}=\mathrm{Ra}^{1 / 2}\left(\nu \kappa \rho_{0} / k\right) p, \quad t=\left(h^{2} / \kappa\right) \tau, \\
& X=h x, \quad Y=h y, \quad Z=h z, \\
& h_{1}=a_{1} / h, \quad h_{2}=a_{2} / h,
\end{aligned}
$$

where $^{\mathbf{5}}$

$$
\kappa=K_{m} / c \rho_{0}, \quad \beta=\max \left|\nabla T^{*}\right|, \quad \mathrm{Ra}=k \gamma \beta g h^{2} / \kappa \nu .
$$

From Eq. (4),

thus,

$$
\nabla T^{*}=\left[\left(T_{1}-T_{0}\right) / h\right] \mathrm{k},
$$

$$
\beta=\left(T_{1}-T_{0}\right) / h, \quad \operatorname{Ra}=k \gamma\left(T_{1}-T_{0}\right) g h / \kappa \nu,
$$

with

$$
\operatorname{Pr}=k \kappa / \nu h^{2} \epsilon, \quad H=(c \rho)_{m} / c \rho_{0}
$$

and $\nabla$ now defined by

$$
\nabla=\left(\frac{\partial}{\partial x}, \frac{\partial}{\partial y}, \frac{\partial}{\partial z}\right)
$$

the dimensionless disturbance equations are therefore

$$
\begin{gathered}
\operatorname{Pr} \frac{\partial \nabla}{\partial \tau}=-\operatorname{Ra}^{1 / 2} \theta \mathbf{k}-\nabla p-\nabla \\
H \frac{\partial \theta}{\partial \tau}+\operatorname{Ra}^{1 / 2} \nabla \cdot \nabla \theta+\operatorname{Ra}^{1 / 2} \nabla \cdot \mathbf{k}=\nabla^{2} \theta \\
\nabla \cdot \nabla=0
\end{gathered}
$$

with

$$
\begin{aligned}
& \mathbf{v} \cdot \mathbf{n}=0 \quad \text { on } x= \pm \frac{1}{2} h_{1}, \quad y= \pm \frac{1}{2} h_{2}, \quad z=0,1, \\
& \theta=0 \quad \text { on } z=0,1 \text {, } \\
& \frac{\partial \theta}{\partial n}=0 \quad \text { on } x= \pm \frac{1}{2} h_{1}, \quad y= \pm \frac{1}{2} h_{2} .
\end{aligned}
$$

There are two methods that may be applied to investigate the stability of disturbances governed by these equations: the linear method and the energy method.

\section{THE LINEAR METHOD}

Here, we assume that the disturbance $(\nabla, \theta)$ is small enough for second-order terms to be neglected. Thus, Eq. (13) reduces to

$$
H \frac{\partial \theta}{\partial \tau}=-\mathrm{Ra}^{1 / 2} \boldsymbol{\nabla} \cdot \mathbf{k}+\nabla^{2} \theta
$$

The other Eqs. (12) and (14) are unaltered.

In the same manner as Davis, ${ }^{6}$ we can write Eqs. (12) and (16) as a vector equation which includes a selfadjoint matrix operator, thereby guaranteeing that the onset of convection is characterized by monotonic growth (the principle of exchange of stabilities). Hence, the stability boundary is determined by the marginal stability equations:

$$
\begin{aligned}
R^{1 / 2} \mathbf{v} \cdot \mathbf{k} & =\nabla^{2} \theta, \\
R^{1 / 2} \theta \mathbf{k}+\mathbf{v} & =-\nabla p,
\end{aligned}
$$

together with (14) and the boundary conditions (15). The critical Rayleigh number $\left(\mathrm{Ra}_{i}\right)_{l \text { in }}$, according to this linear theory, is the smallest eigenvalue of this problem. This critical Rayleigh number, however, gives only a necessary condition for stability, and, therefore,

$$
\left(R a_{c}\right)_{\text {lin }} \geq\left(R a_{c}\right)_{\text {true }} \text {, }
$$

where $\left(\operatorname{Ra}_{c}\right)_{\text {true }}$ is the true critical Rayleigh number. 


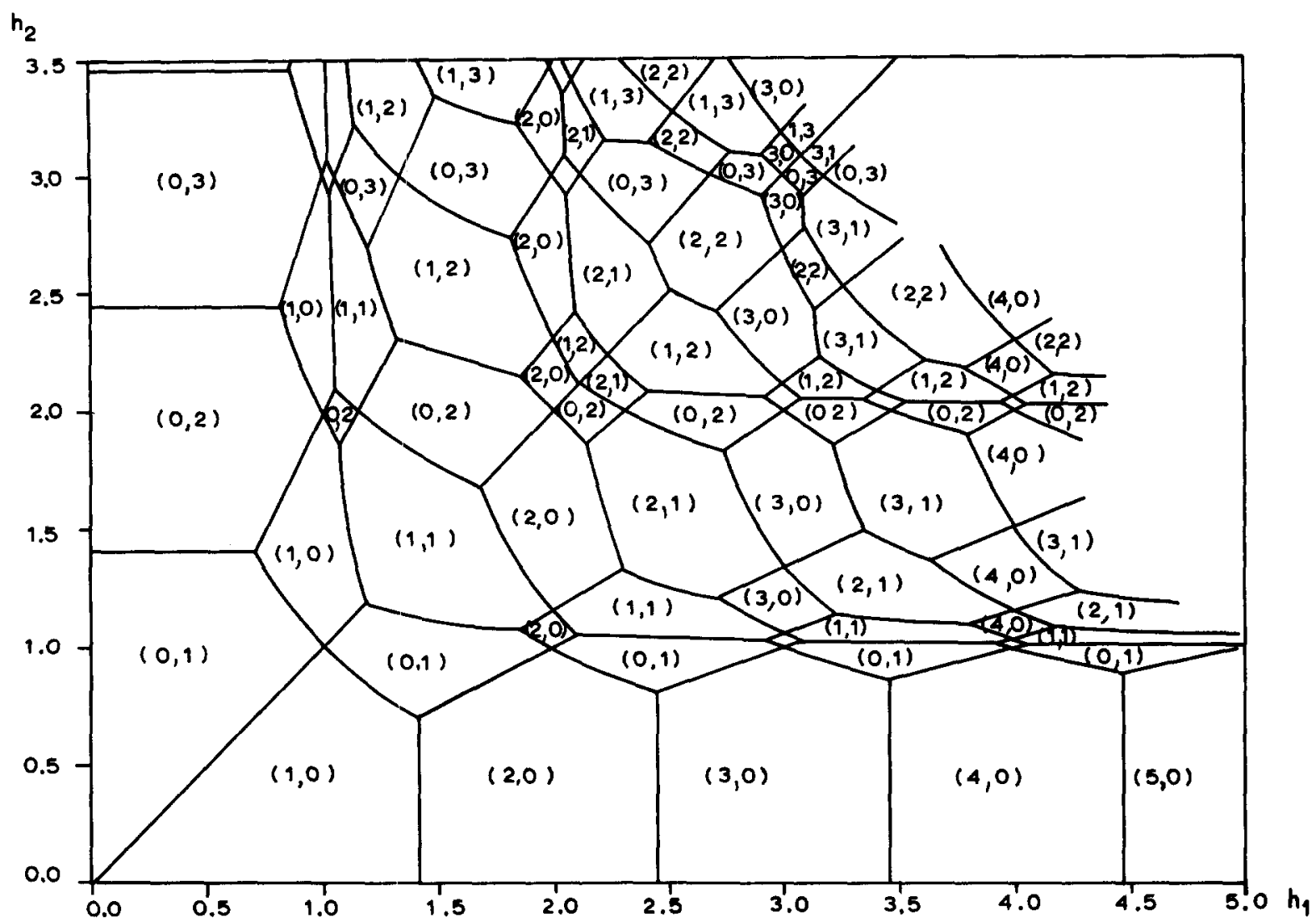

FIG. 2. Preferred cellular mode, $(m, n)$, as a function of $h_{1}, h_{2}$. The figure is symmetric with respect to the line $h_{1}=h_{2}$. The change between the rolls $(m, 0)$ and $(m+1,0)$ occurs at $h_{1}=[m(m+1)]^{1 / 2}$.

\section{THE ENERGY METHOD}

Here, any disturbance $(\nabla, \theta)$ satisfying the continuity equation (14), the boundary conditions (15), and belonging to a class of suitably differentiable functions is admissible. The full equations (12) and (13) are utilized.

The method was adapted for flow in a porous medium by Westbrook. ${ }^{3}$ Both Westbrook's theory and its subsequent extension and modification by Wankat and Schowalter ${ }^{7}$ require the nonlinear inertia term $q \cdot \nabla q$ to be included in Darcy's law for a general theory to be available. This term is specifically required to take into account any kinetic energy being transferred over the boundaries. As it appears that the inclusion of this term leads to an underspecified flow problem (see Appendix A), the analysis carried out in this paper has been restricted to the case in which there is no kinetic energy transferred over the boundaries, that is, there is no normal component of velocity at any boundary. In this event the energy method is fully applicable to the assumed "reduced" Eq. (1) and an analysis parallel to that of Westbrook can be carried out [starting from the disturbance equations (12), (1.3), and (14)] except that his requirement that the energy decrease monotonically [Westbrook, ${ }^{3}$ Eq. (25)] does not insure asymptotic stability in the mean and must be replaced by a theorem analogous to theorem I of Joseph. ${ }^{8}$ This analysis leads to the critical Rayleigh number being defined by the smallest eigenvalue of Eqs. (17) and (18) under the same conditions, satisfaction of continuity (14), and the boundary conditions (15), as in the linear method. Thus, the same eigenvalue problem applies for both approaches.

In this case, however, if $\left(\mathrm{Ra}_{c}\right)_{\mathrm{en}}$ is the critical Rayleigh number given by the energy method

$$
\left(\mathbf{R a}_{c}\right)_{\mathrm{en}} \leq\left(\mathbf{R a}_{c}\right)_{\text {true, }}
$$

since in the energy method one establishes stability relative to arbitrary disturbances subject only to the equation of continuity and the appropriate boundary conditions. For an exact stability criterion, we need only establish stability relative to a subclass of these disturbances, viz., those that satisfy the hydrodynamical disturbance equations. Figure 1 is a schematic diagram of the situation. have

For the problem being considered in this paper, we

$$
\left(\mathrm{Ra}_{c}\right)_{\mathrm{en}}=\left(\mathrm{Ra}_{c}\right)_{\text {lin }},
$$

implying that the common value obtained is the true critical Rayleigh number for the system. 


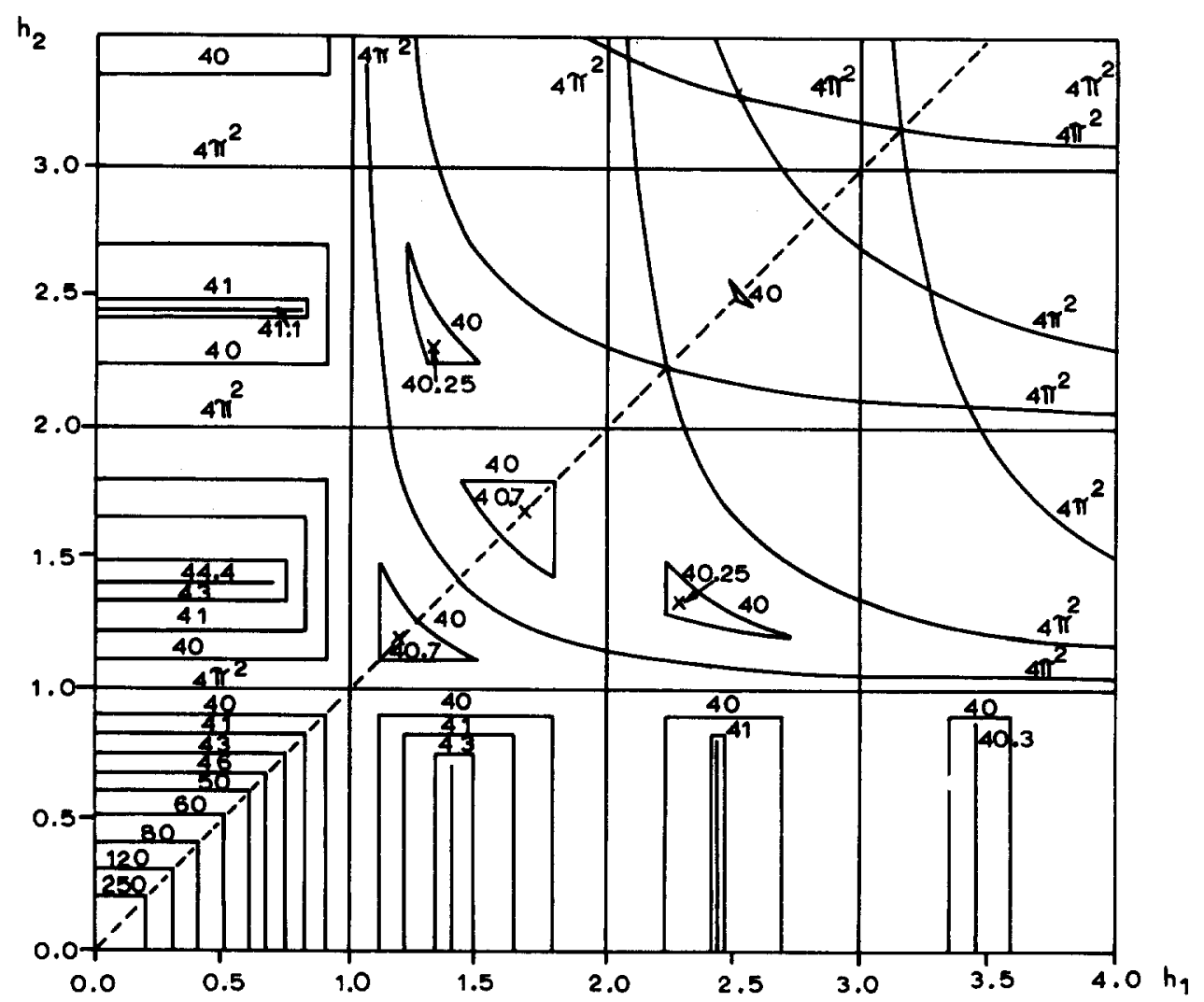

FIG. 3. Variation of $\mathrm{Ra}_{c}$ with $h_{1}, h_{2}$. As either $h_{1}$ or $h_{2}$ becomes large, $\mathrm{Ra}_{c}$ quickly approaches $4 \pi^{2}$, the value for a horizontally unbounded saturated porous medium.

\section{SOLUTION OF THE EIGENVALUE PROBLEM}

Let $\nabla=\left(v_{1}, v_{2}, v_{3}\right)$ and eliminate $p$ from $(18)$, then we have

$$
\begin{gathered}
\frac{\partial v_{3}}{\partial y}-\frac{\partial v_{2}}{\partial z}+R^{1 / 2} \frac{\partial \theta}{\partial y}=0 \\
\frac{\partial v_{3}}{\partial x}-\frac{\partial v_{1}}{\partial z}+R^{1 / 2} \frac{\partial \theta}{\partial x}=0 \\
\frac{\partial v_{2}}{\partial x}-\frac{\partial v_{1}}{\partial y}=0
\end{gathered}
$$

Equations (14) and (17) give, respectively,

$$
\frac{\partial v_{1}}{\partial x}+\frac{\partial v_{2}}{\partial y}+\frac{\partial v_{3}}{\partial z}=0, \quad R^{1 / v_{3}}=\nabla^{2} \theta
$$

The boundary conditions (15) become

$$
\begin{array}{cc}
v_{1}=0 & \text { on } x= \pm \frac{1}{2} h_{1}, \\
v_{2}=0 & \text { on } y= \pm \frac{1}{2} h_{2}, \\
v_{3}=0 & \text { on } z=0,1, \\
\frac{\partial \theta}{\partial x}=0 & \text { on } x= \pm \frac{1}{2} h_{1}, \\
\frac{\partial \theta}{\partial y}=0 & \text { on } y= \pm \frac{1}{2} h_{2}, \\
\theta=0 & \text { on } z=0,1 .
\end{array}
$$

This eigenvalue problem has separable eigenfunctions of the form

$$
\begin{gathered}
v_{1}=\sin \left(\frac{1}{2} m \pi\right)\left[1+\left(2 x / h_{1}\right)\right] \cos \left(\frac{1}{2} n \pi\right)\left[1+\left(2 y / h_{2}\right)\right] U(z), \\
v_{2}=\cos \left(\frac{1}{2} m \pi\right)\left[1+\left(2 x / h_{1}\right)\right] \sin \left(\frac{1}{2} n \pi\right)\left[1+\left(2 y / h_{2}\right)\right] V(z), \\
v_{3}=\cos \left(\frac{1}{2} m \pi\right)\left[1+\left(2 x / h_{1}\right)\right] \cos \left(\frac{1}{2} n \pi\right)\left[1+\left(2 y / h_{2}\right)\right] W(z), \\
\theta=(\pi b)^{-1} \cos \left(\frac{1}{2} m \pi\right)\left[1+\left(2 x / h_{1}\right)\right] \\
\quad \times \cos \left(\frac{1}{2} n \pi\right)\left[1+\left(2 y / h_{2}\right)\right] \Theta(z)
\end{gathered}
$$

where $m, n=0,1,2, \cdots$ but $m+n \neq 0$ and

$$
b=\left[\left(m^{2} / h_{1}^{2}\right)+\left(n^{2} / h_{2}^{2}\right)\right]^{1 / 2} .
$$

We now have the pair of ordinary differential equations:

$$
\begin{aligned}
& W^{\prime \prime}-\pi^{2} b^{2} W-R^{1 / 2} \pi b \Theta=0, \\
& \Theta^{\prime \prime}-\pi^{2} b^{2} \Theta-R^{1 / 2} \pi b W=0,
\end{aligned}
$$

with

$$
\Theta(z)=0=W(z) \quad \text { at } z=0,1 .
$$

The eigenfunctions of the problem (25), (26), and (27) are

$$
\Theta(z)=\sin l \pi z=W(z)
$$

with corresponding eigenvalues

$$
R=\pi^{2}\left[b+\left(l^{2} / b\right)\right]^{2} .
$$


The critical Rayleigh number is, therefore,

$$
\begin{aligned}
\operatorname{Ra}_{c} & =\pi^{2} \min _{(l, m, n)}\left[b+\left(l^{2} / b\right)\right]^{2} \\
& =\pi^{2} \min _{(m, n)}\left(b+b^{-1}\right)^{2} .
\end{aligned}
$$

This minimization problem over the set of pairs of nonnegative integers has been solved with the complementary use of numerical and analytical techniques. The pertinent value of $m$ and $n$ for the minimum eigenvalue depends directly on $h_{1}$ and $h_{2}$. The relationship is illustrated in Fig. 2. This diagram can also be interpreted as giving, for a particular $\left(h_{1}, h_{2}\right)$, the preferred cellular mode for the convective circulation at Rayleigh numbers just above the critical value.

Figure 3 exhibits the dependence of $\mathrm{Ra}_{c}$ on the box geometry parameters $h_{1}$ and $h_{2}$. It can be seen from (29) that the absolute minimum of $\mathrm{Ra}_{c}$ is $4 \pi^{2}$ (given by $b=1$ ), the value obtained by Lapwood ${ }^{4}$ for a horizontally unbounded fluid layer in a porous medium heated from below. Thus, bounding the fluid tends to make it more stable.

\section{DISCUSSION}

The above analysis has shown that for this particular problem the critical Rayleigh numbers given by the linear method and the energy method coincide. Thus, the inequalities (19) and (20) become equalities and subcritical (sublinear) instabilities cannot exist. This conclusion must also apply to the stability of any system featuring a motionless fluid which is contained in a porous medium of arbitrary shape and "heated from below", ${ }^{2}$ provided any bounding surface is both rigid and impermeable. For in this case, the basic state $\left(q^{*}, T^{*}\right)$ is described by $q^{*}=(0,0,0)$ and $T^{*}=\beta Z+T_{0}$ where the $Z$ axis is taken to be in the direction of the gravitational field, $\beta$ is the (constant) temperature gradient throughout the fluid, and $T_{0}$ is the temperature at $Z=0$. The situation is, therefore, similar to that discussed in the analysis above and the eigenvalue problems resulting from the application of the linear and energy methods will be identical. The differential equations of this common eigenvalue problem will be the same as (14), (17), and (18) and these must be solved subject to the flow boundary condition $\nabla \cdot n=0$ on all bounding surfaces and a temperature boundary condition on any bounding surface which is consistent with the basic temperature distribution $T^{*}$.

It is of interest to note that it is only in very narrow, tall boxes that the lateral walls have much effect on the critical Rayleigh number (see Fig. 3). This lack of influence of the walls on $\mathbf{R a}_{c}$ is to be expected since, unlike the corresponding case of a continuous fluid, ${ }^{8}$ there is no viscous dissipation at the lateral walls. The only effect of the walls is thus a geometric one by which those cellular modes which give an approximately square cross section tend to be selected so that there will be an approximate balance between release of potential energy and of viscous dissipation in each cell. ${ }^{6}$
If we define a finite roll as a cell with only two nonzero velocity components, we see that the cellular modes $(m, 0)$ and $(0, n)$ correspond to finite rolls parallel to the $y$ axis and $x$ axis, respectively. However, contrary to the behavior of a continuous fluid where the preferred mode just after the onset of convection is some number of finite rolls with axes parallel to the shorter side, ${ }^{6}$ we find that in a porous medium rolls are invariably preferred whenever the height is not the smallest dimension but that this is no longer the case when the height is less than both lateral dimensions. (Davis ${ }^{6}$ has a linear temperature distribution specified on his lateral walls rather than insulating walls as in this study. However, since viscous dissipation at the walls and the geometry of the box are the dominating influence on the cellular mode, it was felt that the results obtained by Davis would give at least a qualitative description of the cellular behavior of a continuous fluid bounded laterally by insulating walls.) Furthermore, when rolls do form, they are not necessarily parallel to the shorter side. The overriding rule for the formation of rolls appears to be for the number of rolls and the direction of their axes to be such that each roll has the closest approximation to a square cross section possible. Thus for $h_{1}=2.75$ and $h_{2}=0.75$, three rolls form parallel to the $y$ axis whereas an increase of $h_{2}$ to 1.0 results in a single roll parallel to the $x$ axis.

To compare the results of this analysis with the linear analysis of Sutton ${ }^{10}$ for the stability of a quiescent fluid in a bounded two-dimensional porous medium heated from below, we must first decide which special case of the three-dimensional system actually corresponds to the two-dimensional motion considered by Sutton. The only possible conclusion is that motion restricted to the $(x, z)$ plane must correspond to motion in a cross section perpendicular to the axis of a roll which lies parallel to the $y$ axis, i.e., the three-dimensional system with $n=0$. In this case, from (24):

so that (29) gives

$$
b=m / h_{1}, \quad m=1,2, \cdots,
$$

$$
\mathrm{Ra}_{\mathrm{c}}=\pi^{2} \min _{m}\left[\left(m / h_{1}\right)+\left(h_{1} / m\right)\right]^{2}
$$

which is the critical value obtained by Sutton. The value of $m$ given by Sutton for this minimum

$$
m=\text { integral part of }\left[\frac{1}{2}+\frac{1}{2}\left(1+4 h_{1}{ }^{2}\right)^{1 / 2}\right]
$$

agrees with the analysis in this study where it is found that the change from $m$ rolls to $m+1$ rolls occurs at $h_{1}=[m(m+1)]^{1 / 2}$ for $h_{2}$ small. (The pertinent value of $h_{2}$ is some small value since if we look upon the motion considered by Sutton as a special case of the threedimensional system, we see that she is effectively assuming that the $n=0$ mode occurs for all $h_{1}$. From Fig. 2, this is only possible if $h_{2}$ is small.)

As shown in Appendix A, the nonlinear inertia term of the form used by Lapwood ${ }^{4}$ and others working on instability of fluids in porous media is not acceptable in Darcy's law. This means that the energy method in its 
present form cannot be applied to porous medium systems into which fluid is being injected and removed through the walls. At present, an attempt is being made to modify this method to suit such conditions. Indications are that in some cases of interest, $\left(\mathrm{Ra}_{c}\right)_{\text {en }}$ and $\left(\mathrm{Ra}_{c}\right)_{1 \text { in }}$ will not be equal as they have been found to be in this impermeable wall case.

\section{ACKNOWLEDGMENT}

The author wishes to thank Dr. I. G. Donaldson for his helpful suggestions during preparation of the manuscript.

\section{APPENDIX A: CORRECTION TO DARCY'S LAW}

It seems that it has not previously been realized that including the term $\mathbf{q} \cdot \boldsymbol{\nabla q}$ in Darcy's law raises the order of the equation in such a way that the specification of only the normal component of velocity on the boundary leads, in general, to an underspecified system, whereas the specification of the velocity distribution on the boundary gives an overspecified system (that is, in general, it is not possible to satisfy all the boundary conditions). This difficulty has not been noticed previously because those authors (Lapwood, ${ }^{4}$ Nield, ${ }^{1}$ etc.) including the term in Darcy's law have usually applied a linear analysis to the stability of a motionless fluid. In this case the contributions from the nonlinear term vanish. This may be seen in Lapwood's linear analysis ${ }^{4}$ of the stability of a horizontally unbounded layer of quiescent fluid in a porous medium heated from below, where he obtains a fourth-order ordinary differential equation with four boundary conditions because the contributions from the $q \cdot \nabla q$ term vanish. However, if one considers the linear stability of a constant vertical upflow through Lapwood's system, a contribution from the non-linear term remains, producing a fifth-order ordinary differential equation which is subject to only four boundary conditions. Specifically, if we let $W^{*}$ be the magnitude of the upflow, the stability of the basic state $\left(\mathbf{q}^{*}, T^{*}\right)$,

$$
\begin{gathered}
\mathbf{q}^{*}=\left(0,0,-W^{*}\right), \\
T^{*}=\frac{T_{1}-T_{0} \exp \left(-W^{*} h / \kappa\right)+\left(T_{0}-T_{1}\right) \exp \left(-W^{*} Z / \kappa\right)}{1-\exp \left(-W^{*} h / \kappa\right)}
\end{gathered}
$$

to a small disturbance $\left(\mathbf{q}^{\prime}, T^{\prime}\right)$ is to be examined. (The fluid saturating the porous medium is confined between two horizontal, perfectly conducting, rigid planes at $Z=0$ and $Z=h$. The $Z$ axis is vertically downward and the upper and lower boundaries are at temperatures $T_{0}$ and $T_{1}$, respectively.)

Under the Boussinesq approximation, the governing equations are taken to be the "modified" Darcy's law:

$$
\epsilon^{-1} \frac{\partial q}{\partial t}+\frac{1}{\epsilon^{2}} \mathbf{q} \cdot \nabla \mathbf{q}=\left[1-\gamma\left(T-T_{0}\right)\right] g \mathbf{k}-\rho_{0}^{-1} \nabla p-\frac{\nu}{k} \mathbf{q},
$$

The linearized equations governing the disturbance are

$$
\begin{gathered}
\epsilon^{-1} \frac{\partial \mathbf{q}^{\prime}}{\partial t}-\frac{1}{\epsilon^{2}} W^{*} \frac{\partial \mathbf{q}^{\prime}}{\partial Z}=-\gamma g T^{\prime} \mathbf{k}-\rho_{0}^{-1} \nabla p^{\prime}-\frac{\nu}{k} \mathbf{q}^{\prime}, \\
(c \rho)_{m} \frac{\partial T^{\prime}}{\partial t}+c \rho_{0} \mathbf{q}^{\prime} \cdot \nabla T^{*}-c \rho_{0} W^{*} \frac{\partial T^{\prime}}{\partial Z}=K_{m} \nabla^{2} T^{\prime}, \\
\nabla \cdot q^{\prime}=0,
\end{gathered}
$$

with

$$
\mathbf{q}^{\prime} \cdot \mathbf{n}=0 \text { and } T^{\prime}=0 \text { at } Z=0, h \text {. }
$$

These equations and the boundary conditions are reduced to dimensionless form by the substitutions (9), (10), and (11) together with

From (A1)

$$
\alpha=W^{*} h / \kappa \text {. }
$$

$$
\nabla T^{*}=\frac{\left(T_{1}-T_{0}\right)\left(W^{*} / \kappa\right)}{1-\exp \left(-W^{*} h / \kappa\right)} \exp \left(-\frac{W^{*} Z}{\kappa}\right) \mathbf{k} .
$$

Thus,

$$
\beta=\max \left|\nabla T^{*}\right|=\frac{\left(T_{1}-T_{0}\right)\left(W^{*} / \kappa\right)}{1-\exp \left(-W^{*} h / \kappa\right)} .
$$

The dimensionless disturbance equations are, therefore,

$$
\begin{gathered}
\operatorname{Pr} \frac{\partial \boldsymbol{v}}{\partial \tau}-\operatorname{Pr} \frac{\alpha}{\epsilon} \frac{\partial \mathbf{v}}{\partial z}=-\operatorname{Ra}^{1 / 2} \theta \mathbf{k}-\nabla p-\mathbf{v} \\
H \frac{\partial \theta}{\partial \tau}-\alpha \frac{\partial \theta}{\partial z}=-\operatorname{Ra}^{1 / 2} e^{-\alpha z} \mathbf{v} \cdot \mathbf{k}+\nabla^{2} \theta \\
\nabla \cdot \mathbf{v}=0
\end{gathered}
$$

with

$$
\nabla \cdot n=0 \text { and } \theta=0 \text { at } z=0,1 \text {. }
$$

Let $\mathbf{v}=\left(v_{1}, v_{2}, v_{3}\right)$ and eliminate $p$ from (A5) using (A7), then

$$
\left[\operatorname{Pr} \frac{\partial}{\partial \tau}-\operatorname{Pr} \frac{\alpha}{\epsilon} \frac{\partial}{\partial z}+1\right] \nabla^{2} v_{3}=-\operatorname{Ra}^{1 / 2} \nabla_{1}^{2} \theta
$$

where

$$
\nabla_{1}^{2}=\frac{\partial^{2}}{\partial x^{2}}+\frac{\partial^{2}}{\partial y^{2}}
$$

From (A6), we get

$$
\left[H \frac{\partial}{\partial \tau}-\alpha \frac{\partial}{\partial z}-\nabla^{2}\right] \theta=-\mathrm{Ra}^{1 / 2} e^{-\alpha v_{v_{3}}} .
$$

Let

$$
\left[\theta, v_{3}\right]=e^{\sigma \tau} f(x, y)[\Theta(z), W(z)],
$$

where $\nabla_{1}^{2} f=-a^{2} f$. Let $D \equiv d / d z$, then substituting (A11) in (A9) and (A10), we get

$$
\begin{array}{r}
{\left[\operatorname{Pr} \sigma-\operatorname{Pr} \frac{\alpha}{\epsilon} D+1\right]\left(D^{2}-a^{2}\right) W=\operatorname{Ra}^{1 / 2} a^{2} \Theta} \\
{\left[H \sigma-\alpha D-\left(D^{2}-a^{2}\right)\right] \Theta=-\mathbf{R a}^{1 / 2} e^{-\alpha z} W,}
\end{array}
$$

with

$$
\Theta(z)=0=W(z) \quad \text { at } \quad z=0,1 .
$$


Thus, we have a fifth-order ordinary differential equation in $W$ but only four boundary conditions, that is, an underspecified system is obtained. It may be thought that this difficulty could be overcome by introducing the no-slip boundary conditions used in continuous fluid studies. However, this would give the additional conditions

$$
D W(z)=0 \text { at } z=0,1 \text {. }
$$

so that we have six boundary conditions and an overspecified system results.

To obtain a fourth-order ordinary differential equation, we must exclude the term $\operatorname{Pr}(\alpha / \epsilon) D$. This arises from the $\mathbf{q} \cdot \boldsymbol{\nabla q}$ term in the original equations. We therefore conclude that this nonlinear term must be omitted from Darcy's law.

It appears that if any nonlinear inertia term should be included at all, it should be of the form $q \mathrm{q}$ (see, for example, Irmay ${ }^{11}$ ). However, an order of magnitude analysis of the terms in Darcy's law shows that such an inertia term may be neglected, provided the Reynolds number based on the flow through the pores does not exceed $O(1)$. This is satisfied for most cases of interest in the practical applications of stability theory.

1 D. A. Nield, Water Resources Res. 4, 553 (1968).

2 D. D. Joseph, Arch. Ratl. Mech. Anal. 20, 59 (1965).

${ }^{3}$ D. R. Westbrook, Phys. Fluids 12, 1547 (1969).

4 E. R. Lapwood, Proc. Cambridge Phil. Soc. 44, 508 (1948).

${ }^{5}$ It should be noted that $\alpha$ is neither the thermal diffusivity of the fluid nor that of the saturated medium.

$6 \mathrm{~S}$. H. Davis, J. Fluid Mech. 30, 465 (1967).

7 P. C. Wankat and W. R. Schowalter, Phys. Fluids 13, 2418 (1970).

${ }^{8}$ D. D. Joseph, Arch. Ratl. Mech. Anal. 22, 163 (1966).

$\checkmark$ By a fluid heated from below we mean a fluid in which the temperature gradient is in the direction of the gravitational field when the fluid is at rest.

${ }^{10}$ F. M. Sutton, Phys. Fluids 13, 1931 (1970).

${ }^{11}$ S. Irmay, Trans. Am. Geophys. Union 39, 702 (1958).

\title{
Stability of a T'wo-Component Fluid Layer Heated from Below
}

\author{
J. C. Legros \\ Facultê des Sciences Appliquées and Faculte des Sciences, Universite Libre de Bruxelles, Brussels, Belgium \\ AND \\ J. K. Platten \\ Faculte des Sciences, Université de l'Etat, Mons, Belgium \\ AND \\ P. G. Potx \\ Faculte des Sciences, Universite Libre de Bruxelles, Brussels, Belgium
}

(Received 9 October 1970; final manuscript received 21 June 1971)

\begin{abstract}
In connection with recent experimental results, the stability of a two-component fluid layer heated from below is examined taking into account the concentration gradient due to thermal diffusion. With the use of a variational method (local potential technique) developed by Glansdorff and Prigogine, an approximate solution is proposed for dilute solutions. The critical Rayleigh number increases for negative thermal diffusion factors and decreases for positive ones.
\end{abstract}

\section{INTRODUCTION}

The study of the hydrodynamic stability of a thin horizontal layer of fluid heated from below is a classical subject. A detailed analysis of this stability problem can be found in the monograph by Chandrasekhar. ${ }^{1}$ It is shown that the fluid layer at rest becomes unstable when the temperature gradient exceeds a critical value, more precisely when the Rayleigh number, defined as

$$
\mathrm{Ra}=g \alpha \beta d^{4} / K \nu,
$$

exceeds the value 1708 for a liquid layer confined between two rigid boundaries. In (1), $g$ denotes the acceleration due to the gravity, $\alpha$ is the thermal expansion coefficient, $\beta$ is the adverse temperature gradient, $K$ is the thermal diffusivity, $\nu$ is the kinematic viscosity, and $d$ is the thickness of the layer.
This study, however, applies to pure liquid layers. For liquid mixture layers, thermal diffusion (Soret effect) must be taken into account, so we expect a quite different behavior for the fluid layer. Indeed, the density gradient is the result of the thermal expansion of the liquid and also of the mass fraction distribution due to thermal diffusion, if we allow the density to be a function of temperature and mass fraction.

For a positive Soret coefficient $\left(D^{\prime} / D>0\right)$, the more dense component migrates toward the cold plate (upper boundary) and reinforces the "thermal" density gradient. We expect the fluid layer to be less stable than in the pure liquid case. On the contrary, a negative Soret coefficient would produce an opposite effect.

Recently, some experimental results ${ }^{2-4}$ did not completely confirm those crude prediction. Legros et al. observed a stabilizing effect in $\mathrm{H}_{2} \mathrm{O}-\mathrm{CH}_{3} \mathrm{OH}$ and $\mathrm{H}_{2} \mathrm{O}-$ 